\title{
A Study of the Hydrodynamic Performance of a Pitch-type Wave Energy Converter-Rotor
}

\author{
Sunny Kumar Poguluri ${ }^{\circ}$, Il-Hyoung Cho and Yoon Hyeok Bae * \\ Department of Ocean System Engineering, Jeju National University, Jeju, Korea; \\ sunnykumar@jejunu.ac.kr (S.K.P.); cho0904@jejunu.ac.kr (I-H.C.) \\ * Correspondence: yh.bae@jejunu.ac.kr (Y.H.B.); Tel.: +82-64-754-3485
}

\begin{abstract}
The effect of hydrodynamic performance of the wave energy converter (WEC)-rotor based on linear potential flow theory due to nonlinear viscous damping was investigated. Free decay tests were conducted using computational fluid dynamics (CFD) to obtain the viscous damping moment. The commonly used procedure for obtaining the damping moment is based on peak amplitudes which normally require a long time history records. Such long free decay records may not be possible in nodding WEC rotor due high damping. The energy method proposed by Bass and Haddara requires only the short and full range of the recorded data. This method provides sufficiently good results when the bodies have high damping. The method equates the rate of change of the total energy of a body undergoing free rolling/pitching to the rate of energy dissipated by the damping. The present study adopts a similar methodology for estimating the linear and linear plus quadratic damping. To incorporate the nonlinear viscous damping moment in the linear equation of motion, an equivalent linearization concept is used without neglecting the nonlinear damping effects. The hydrodynamic coefficients obtained from the linear potential flow theory, nonlinear viscous damping moment from the energy method and estimated PTO damping are used to solve the equation of motion of the WEC rotor. The estimated pitch free decay data shows good agreement with the simulated CFD results when compared to the linear viscous damping moment and better agreement is obtained with linear plus quadratic viscous damping moment. The regular and irregular wave analyses show that a considerable effect on the hydrodynamic performance of the WEC rotor is observed when the linear and linear plus quadratic viscous damping are included.
\end{abstract}

Keywords: wave energy converter; potential flow theory; CFD; energy method; linear and linear plus quadratic viscous damping; free decay test

\section{Introduction}

It has long been recognized that ocean waves carry vast amounts of energy. This has led to the development of various methods for exploiting waves by converting wave energy into mechanical and electrical energy. Even though different wave energy technologies have been reported in the literature, the vast majority of these are still in the stage of trying to identify the best designs and very few have reached the stage of practical application. Overall, the available wave energy converter (WEC) technologies may be categorized based on the IEA-Ocean Energy Systems as oscillating water columns, wave activated bodies and overtopping. A detailed review and conceptual design of these technologies are provided in [1-3]. In wave-activated bodies, Salter [4] proposed a unique WEC concept where a duck shape bobs up and down in a wave field, achieving efficiencies of almost $90 \%$ in two-dimensional regular wave conditions. This is often referred as Salter's duck. Mei [5] concluded that maximum extraction can be obtained when the WEC and the fluid oscillate at resonance along a single degree of freedom and that the extraction is greater for non-symmetrical bodies. Based on this concept, the 
evolution of Salter's Duck (rotor) to the proposed commercial WEPTOS rotor in Danish North Sea Pt3, EMEC and Yeu Island [6] can be seen in the literature. The development of Salter's duck has been well-documented by the Edinburgh Wave Power Group [7,8]. The essential geometric features of Salter's duck (rotor) are based on an efficient hydrodynamic shape. The WEC rotor consists of a beak, stern and paunch parts as shown in Figure 1. The paunch of the WEC rotor is shaped such that the dynamic pressure caused by the wave-induced water particle motions efficiently forces the duck to rotate about its fixed axis. The wave induced response can be converted into useful energy by the power-take-off (PTO) system of the WEC. Many researchers have expanded the scope of application of the WEC rotor to realistic environmental conditions (see Pecher $[3,9]$ ) in a full scale application. In the present study, the hydrodynamic performance of the WEC rotor based on Salter's duck is assessed in western waters of Jeju Island.

The size of a body in relation to waves to which it is exposed to may be described by the scattering parameter $k a$, where $k$ is the wave number and $a$ is the characteristic dimension of the body [10]. For large $k a$, wave diffraction occurs around a floating body and the body can further radiate waves due to its motion. Bodies with large $k a$, small motions and low steepness can be treated using the linear radiation-diffraction theory in conjunction with the linear wave theory. This subject is fairly well-understood in the literature. Simplified linear potential flow theories always overestimate or underestimate the response of the floating bodies [11,12]. Poguluri and Bae [12] carried out a free decay test on a pitch-type WEC rotor with a draft of $2.8 \mathrm{~m}$ and beak angle of $60^{\circ}$ without and with kinematic viscosity and obtained a difference of $40 \%$. This difference has been attributed to the nonlinear effects and additionally, an unrealistic response amplitude operator (RAO) of the WEC rotor is obtained without viscous damping moment. Bodies such as the WEC rotor are prone to oscillate at large angles of rotation and require nonlinear radiation-diffraction analysis along with the viscous forces. The response of the WEC rotor can be estimated accurately either by modeling the nonlinear time domain wave-body interaction using computational fluid dynamics (CFD) or through weak coupling of the potential-based solution by incorporating the nonlinear external restoring and damping moments. The latter method is faster and gives an appropriate estimate than the former method which are computationally time consuming. The weakly coupled solvers generally require the use of nonlinear restoring and damping moments. Many methods for representing restoring and damping in different nonlinear forms have been discussed in the literature. Dalzell [13] investigated ship rolling based on conventional linear, quadratic and cubic models using slowly varying parameters and the least square technique as a function of damping moments. Robert [14] investigated a general approach to the analysis of decay tests, including nonlinearities for both the damping and restoring terms. He related the parameters of the roll damping moment, including the amplitude of rolling motion, to a loss function using a parametric identification procedure. This method is sensitive to distortions in the roll decrement and this problem is overcome by fitting a spline through the peaks. Mathisen and Prince [15] developed a perturbation solution for the differential equation of the free and forced roll motion to obtain the coefficients in linear models with quadratic and cubic damping moments. This method assumes that the nonlinear response is a small perturbation of the linear response, making the method valid only for small nonlinearities. The above methods require the use of the peak amplitudes only and longtime history records are required to obtain a reliable estimate of the damping moment from the free decay curve. This may not be possible in the nodding WEC rotor [16] when the damping is high. Bass and Haddara [17] introduced an energy method for estimating the roll damping coefficients from the decay data. This method does not use the peak values of the free decay curve for obtaining the rolling moment. Instead, it is based on the concept that the rate of change of the total energy of a body undergoing free rolling equals the rate of the energy dissipation by the damping and utilizes the full range of the recorded decay curve. This method allows the analysis of very short decay records and can be used to estimate the damping coefficients from the decay data with large damping. The derivatives of the decay curves play a vital role in the energy method and these are accompanied by the 'noise' as also reported by Roberts [18]. Therefore, it is necessary to eliminate the noise associated 
with the decay curves. A simplified approach for such noise elimination based on a least-squares fit to the recorded decay data was proposed by Zhang [19], and enabled the identification of the true decay curve. Thus, the noise from the derivatives can be reduced to the large extent. Sun and Sun [20] used partial least-squares regression instead of the traditional least-squares regression in conjunction with the energy method to identify the parameters of nonlinear roll damping [19,21]. Many researchers extended the application of the energy method to different floating vessels such as the trimaran ship [19], semi-submersible [20] and a fishing vessel [21] and estimated the linear and nonlinear viscous roll damping coefficients. To facilitate the incorporation of nonlinear damping into the linear equation of motion, Lloyd [22] used the equivalent linearized roll damping coefficient that has been related to energy dissipation (see [23]). This makes it possible to solve the equation of motion using linear theory including the effects of nonlinear damping coefficients. In the present study, a similar methodology followed by Zhang [19] was adopted to assess the hydrodynamic performance of the WEC rotor by conducting a free decay test using CFD and the linear and linear plus quadratic viscous damping moment coefficients were obtained using the energy method. The estimated coefficients were added as an external damping to solve the equation of motion of the WEC rotor. The method of linear equivalent concept was used to solve the motion equation of the WEC rotor by including the non-linear damping. Section 2 gives details of the theoretical formulations of WEC rotor, outlines the energy method and linear equivalent concept. Section 3 presents the methodology, numerical validation with the experimental results of the WEC rotor. Section 4 deals with the sensitivity of the WEC rotor to the extractable power in regular and irregular waves. Finally Section 5 presents the salient conclusions.

\section{Theory}

A three-dimensional Cartesian coordinate system $(x, y, z)$ was used to analyze the WEC rotor, with the $x$-axis aligned with the wave propagation direction, $z$-axis pointing upward and origin ' $O$ " placed at the center of the axle of rotation in a water depth $(d)$ of $80 \mathrm{~m}$ (see Figure 1). The rotor is $5 \mathrm{~m}$ wide $(W)$ in the y-direction and the basic geometry of the WEC rotor was adopted from the work of Jeffrey et al. [8].

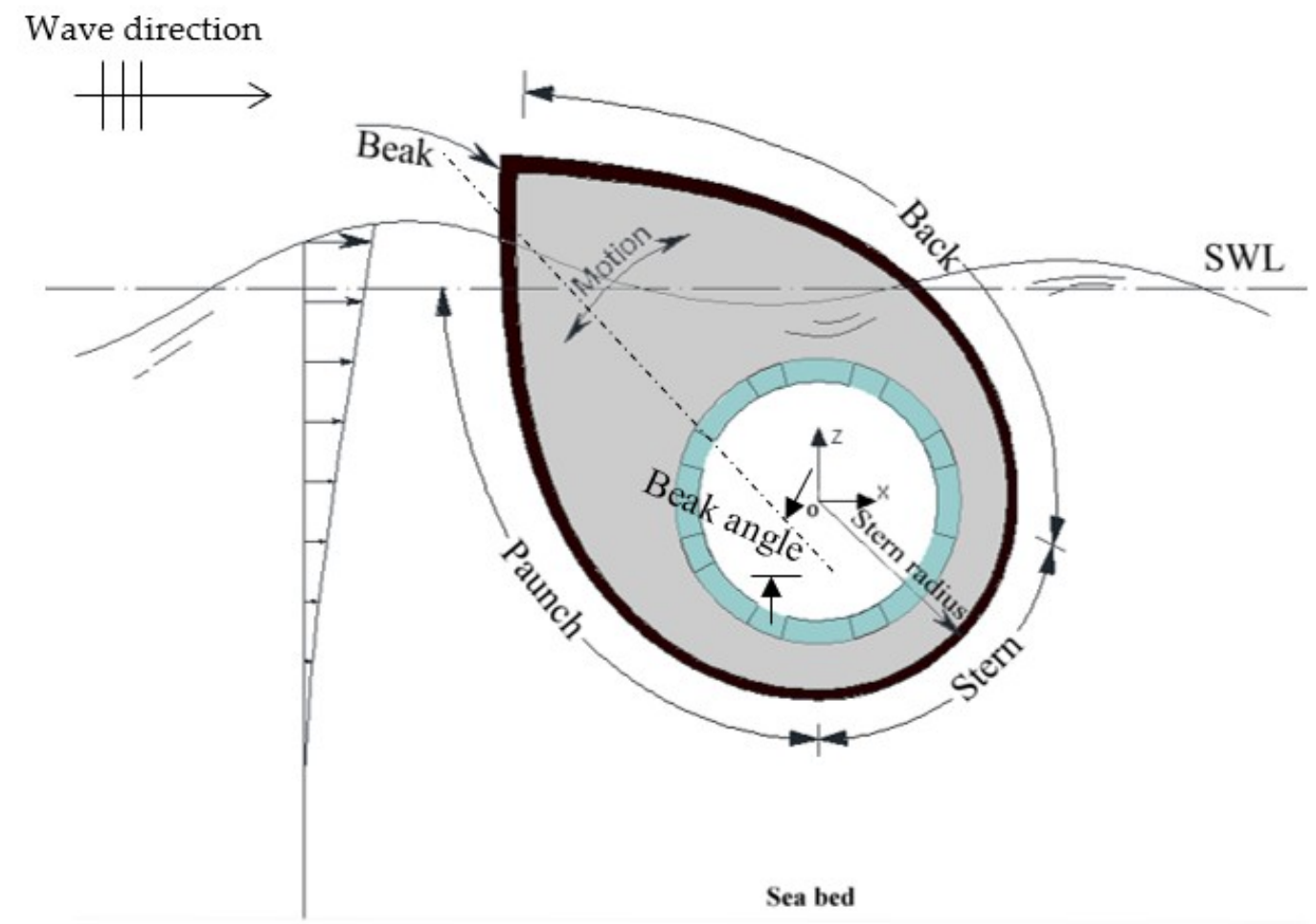

Figure 1. Schematic diagram of a WEC rotor. 
Two rotor placements are analyzed, one with the submergence axis at $0.8 \mathrm{~m}$ below SWL (Rotor 1 ), and one with the submergence axis at $2.2 \mathrm{~m}$ below SWL (Rotor II). Both rotors have the beak angle of $60^{\circ}$. Other important parameters such as center of gravity, inertia and hydrostatic properties are listed in Table 1. The mass and the moments of inertia in the $x, y$ and $z$ direction $\left(I_{x x}, I_{y y}\right.$ and $I_{z z}$ respectively) were calculated using a solid modeling (Solidworks ${ }^{\circledR}$ ) software. The hydrostatic restoring pitch coefficient $\left(K_{55}\right)$ was obtained using the WAMIT software (see Table 1$)$. The WEC rotor has a characteristic length $(l)$ of $1 \mathrm{~m}$. The geometry of the WEC rotor is modeled and discretized using Multi-Surf with 1,400 panels. The rotor is exposed to the wave frequencies ranging from 0.1 to $6 \mathrm{rad} / \mathrm{s}$ and only half of the model was considered for analysis due to the symmetry in the $x z$-plane.

Table 1. Details of the prototype WEC rotor.

\begin{tabular}{ccc}
\hline Description & \multicolumn{2}{c}{ Prototype } \\
\cline { 2 - 3 } & Rotor I & Rotor II \\
\hline Total mass $(\mathrm{kg})$ & 17298 & 38712 \\
$\mathrm{I}_{\mathrm{xx}}$ about Center of Rotation $\left(\mathrm{kg} \cdot \mathrm{m}^{2}\right)$ & 104065 & 211302 \\
$\mathrm{I}_{\mathrm{yy}}$ about Center of Rotation $\left(\mathrm{kg} \cdot \mathrm{m}^{2}\right)$ & 110581 & 209067 \\
$\mathrm{I}_{\mathrm{zz}}$ about Center of Rotation $\left(\mathrm{kg} \cdot \mathrm{m}^{2}\right)$ & 92984 & 172102 \\
Hydrostatic coefficient $\left(\mathrm{K}_{55} / \rho g \mathrm{l}^{4}\right)\left(\mathrm{kg} \cdot \mathrm{m}^{2} \cdot \mathrm{s}^{-2}\right)$ & 12.74 & 41.11 \\
Horizontal center of gravity $\left(\mathrm{X}_{g}\right)(\mathrm{m})$ & -0.6174 & -0.5692 \\
Vertical center of gravity $\left(\mathrm{Z}_{g}\right)(\mathrm{m})$ & 0.7661 & 0.8396 \\
Natural frequency $\left(\omega_{N}\right)(\mathrm{rad} / \mathrm{s})$ & 1.03 & 1.17 \\
\hline
\end{tabular}

Using linear theory, the un-coupled pitch mode of a WEC rotor is described by Equation (1) [24-26]. Here, the components of the rotor mode are the wave exciting moment $F_{5}{ }^{E}(t)$, hydrostatic moment $F_{5} S\left(t, \zeta_{5}\right)$, radiation moment $F_{5}{ }^{R}\left(t, \frac{d \zeta_{5}}{d t}, \frac{d^{2} \zeta_{5}}{d t^{2}}\right)$ and the total damping is from viscous damping moment $F_{5}^{v}\left(t, \frac{d \zeta_{5}}{d t}\right)$ and PTO damping moment $F_{5}{ }^{P T O}\left(t, \frac{d \zeta_{5}}{d t}\right)$ and the full expression is:

$$
M_{55} \frac{d^{2} \zeta_{5}}{d t^{2}}=F_{5}^{E}+F_{5}^{S}+F_{5}^{R}+F_{5}^{V}+F_{5}^{P T O}
$$

where $M_{55}$ is the total inertia, subscript ' 5 ' corresponds to the pitch motion of the WEC rotor and $\frac{d}{d t}$ is the total time derivative. The angular displacement $\xi$ between the coordinate systems is assumed to be a harmonic function of time given by:

$$
\zeta_{5}(t)=\operatorname{Re}\left\{\xi_{5} e^{-i \omega t}\right\}
$$

where $\omega$ is angular frequency of the exciting wave. By substituting, the displacement and corresponding velocity and acceleration of the WEC rotor obtained from Equation (2) into Equation (1) and rearranging, the equation of motion for the WEC rotor in the pitch mode can be written as [24]:

$$
\left|\frac{\xi_{5}}{a}\right|=\frac{\left|X_{5}\right|}{\sqrt{\left[\left(K_{55}-\left(J+A_{55}\right) \omega^{2}\right)^{2}+\left(B_{55}^{\text {rad }}+B_{55}^{\text {vis }}+B_{55}^{\text {PTO }}\right)^{2} \omega^{2}\right]}}
$$

Here, the hydrodynamic coefficients $A_{55}, K_{55}$ and $X_{5}$ are the added mass inertia, hydrostatic stiffness moment and wave excitation moment that are obtained by WAMIT, ' $J$ ' is the mass moment of inertia of the WEC rotor and ' $B_{55}^{r a d}, B_{55}^{v i s}$ and $B_{55}^{P T O '}$ are the radiation, viscous and PTO damping moments.

The optimal capture width is given by:

$$
l_{o p t}=\frac{\bar{P}_{o p t}}{\frac{1}{2} \rho g A^{2} C_{g}}
$$


where:

$$
\bar{P}_{o p t}(\omega)=\frac{1}{2} \omega^{2} \widetilde{B}_{55}^{P T O} a^{2}\left|\frac{\xi_{5}}{a}\right|^{2}
$$

and $C_{g}$ is the group celerity given by $\frac{C}{2}\left[1+\frac{2 k d}{\sinh (2 k d)}\right]$, ' $C$ ' is the celerity of the wave, ' $k$ ' is the wave number and ' $d$ ' is the water depth and the optimal power-take-off $\left(\widetilde{B}_{55}^{P T O}\right)$ coefficient is:

$$
\widetilde{B}_{55}^{P T O}(\omega)=\frac{\sqrt{\left(\omega_{N}^{2}-\omega^{2}\right)^{2}\left(J+A_{55}\right)^{2}+\omega^{2}\left(B_{55}^{r a d}+B_{55}^{v i s}\right)^{2}}}{\omega}
$$

For irregular wave analysis, WEC operating in the western coast of Jeju waters located at an latitude of 33,21,05 and longitude 126,06,18 in Korea with a water depth of $80 \mathrm{~m}$ is considered. With the available wave measured data, the Joint North Sea Wave Project (JONSWAP) wave spectrum is chosen for the analysis. The pitch motion response spectrum for the WEC rotor can be obtained by the transfer function of the pitch motion and the wave spectrum:

$$
S_{\xi_{5}}(\omega)=\left|\frac{\xi_{5}(\omega)}{a}\right|^{2} \cdot S_{\zeta}(\omega)
$$

and the $S_{\zeta}(\omega)$ is given by the JONSWAP spectrum as given by:

$$
S_{\zeta}(\omega)=\alpha_{s} \frac{H_{1 / 3}^{2} \omega_{P}^{4}}{\omega^{5}} \exp \left[-1.25\left(\frac{\omega}{\omega_{P}}\right)^{-4}\right] \gamma^{\beta_{s}}
$$

with:

$$
\begin{aligned}
\omega_{P} & =\frac{2 \pi}{T_{P}} \\
\alpha_{S} & =\frac{0.0624}{0.23+0.0336 \gamma-0.185(1.9+\gamma)^{-1}}(1.094-0.01915 \ln \gamma) \\
\beta_{S} & =\exp \left[-\frac{\left(\omega-\omega_{P}\right)^{2}}{2 \sigma^{2} \omega_{P}^{2}}\right]
\end{aligned}
$$

where $\gamma$ is the peakedness factor, $T_{P}$ is the spectral peak period, $H_{1 / 3}$ is the significant wave height and the value of the spectral width parameter $\sigma$ depends on the frequency according to:

$$
\sigma= \begin{cases}0.07 & \omega<\omega_{P} \\ 0.09 & \omega>\omega_{P}\end{cases}
$$

Finally, the square root of the power spectrum can be estimated as the product of the mean optimal power and the response spectrum and is given by Equation (9):

$$
S_{\sqrt{\bar{P}}}(\omega)=\frac{\bar{P}_{o p t}(\omega)}{a^{2}} S_{\zeta}(\omega)
$$

The square root of the average extracted power can be written as:

$$
\bar{P}_{i r r}=\int_{0}^{\infty} S_{\sqrt{\bar{p}}}(\omega) d \omega
$$

In the present paper, the power analysis is carried out based on the frequency domain solution only so that variable optimal PTO damping is used to obtain the final square root of the power spectral response. It is more appropriate to use the single PTO damping in the time domain analysis rather than in frequency domain analysis. To obtain the damping coefficients from the free decay time history, the energy method is used. The present authors previously described the energy method in detail [16] 
and this approach is also outlined in Appendix A. This method can be applied to one or more cycles of the decay curve to attain the sufficient accuracy. The most common method for obtaining the damping is using the linear, linear plus quadratic and linear plus cubic damping. In the present formulation, linear and linear plus quadratic damping is investigated.

To incorporate the nonlinear viscous damping moment in the linear equation of motion, Lloyd [22] proposed the equivalent linearization concept, allowing the continued use of the linear equation of motion including the effects of nonlinear damping moment coefficients and the same has been used, as can be expressed by Equation (11) which contains the initial pitch angle $\xi_{50}$ that is assumed in the calculation of $B_{55(e q)}$ :

$$
B_{55(e q)}=B 1_{55}+\frac{8}{3 \pi} B 2_{55} \xi_{50} \omega
$$

\section{Method}

The wave exciting forces or moments can be categorized into different regimes as a function of $\pi D / \lambda$ and $H / D$ where ' $D$ ' is the characteristic dimension, ' $H$ ' is the wave height and ' $\lambda$ ' is the wave length. For small bodies $\pi D / \lambda<<1$ and it augments the effect of inertia and the drag forces as shown in Figure 2. If ' $D$ ' is larger than the ' $\lambda$ ', the body is regarded as large, and its presence significantly alters the wave propagation pattern and gives rise to diffraction. We note that the chosen rotors exposed to different wave frequencies are found in a variety of different regimes marked by $(+)$ in Figure 2. Both the diffraction and viscous effects may be important for determining the wave-induced motions of the WEC rotor. Therefore, the final response of the WEC rotor was obtained based on the linear radiation/diffraction method and by incorporating the nonlinear viscous moment as an external damping.

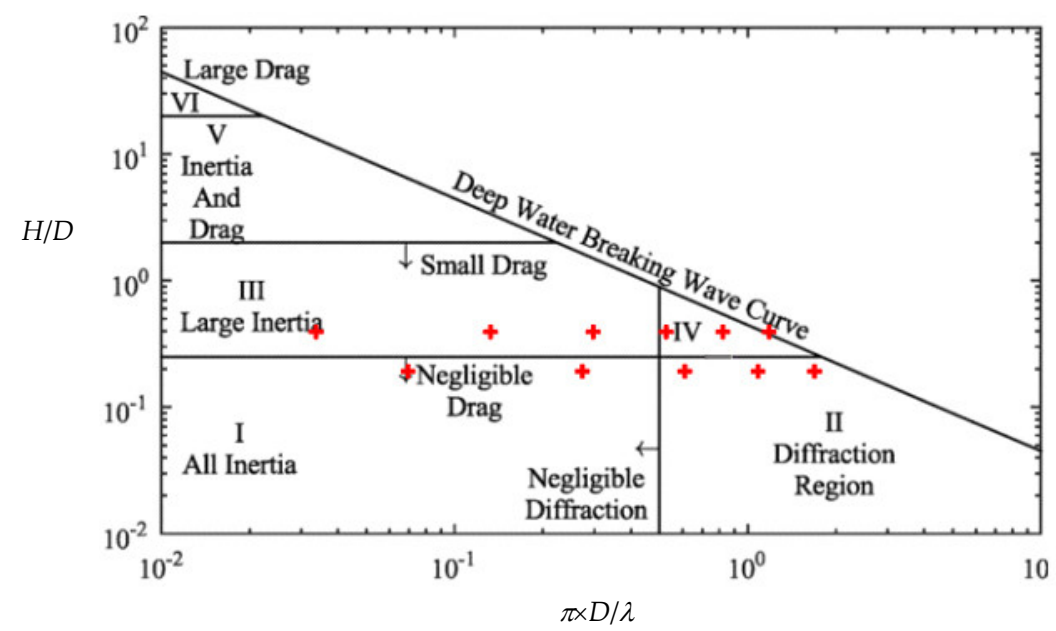

Figure 2. Different wave force regimes exposed to the WEC rotor [10]; (+) represents the WEC rotors exposed to different wave frequencies in the investigation.

\subsection{Physical Experiments}

Numerical validation was first performed on the WEC model scale and then, the prototype WEC rotor was solved applying the same procedure. The performance assessment of the prototype WEC rotors with two different configurations as detailed in Table 1 was carried out using the linear potential flow theory, including the nonlinear damping by conducting free decay test with the incompressible turbulent model based on the Reynolds-averaged Navier-Stokes (RANS) equations in STAR-CCM+.

Numerical validation in the free decay test was carried out by comparing to the experimental results obtained for the model scale WEC rotor. The principal parameters of the prototype and model of a WEC rotor are given in Table 2. The experiments were conducted in the wave flume $(20 \mathrm{~m} \times 0.8 \mathrm{~m} \times 1.0 \mathrm{~m})$ at the Department of Ocean System Engineering, Jeju National University. One 
end of the flume has a piston-type wave maker and the other end has a wave absorber. The rotor is placed at a distance of $8.5 \mathrm{~m}$ from the wave maker (Figure 3) and water depth is maintained at $0.6 \mathrm{~m}$. A WEC rotor was fabricated in the 1:14.8 scale using acrylic material. The model is allowed to move up and down about its center of rotation (COR) by the means of a shaft that passes through the rotor center, and both ends of the shaft are connected to vertical rods that are fixed with the help of clamps in the wave flume. To reduce the frictional force, a bearing mechanism is used between the shaft and vertical rods that allows the rotor to pitch smoothly about the COR. The rotor can be fixed at the required draft by moving the vertical rods. A schematic drawing of the test facilities and the experimental model setup are presented in Figure 3.

Table 2. Prototype and model details (Scale 1:14.8).

\begin{tabular}{ccc}
\hline Description & Prototype & Model Scale \\
\hline Mass $(\mathrm{kg})$ & 42509 & 12.74 \\
$\mathrm{CG}(\mathrm{m}) \mathrm{x}, \mathrm{y}$, and z coordinates (from SWL) & $-1.480,0,1.48$ & $-0.0951,0,0.0957$ \\
Draft $(\mathrm{m})$ & 4.2 & 0.29 \\
Beak angle $(\mathrm{deg})$ & 36 & 36 \\
$\mathrm{I}_{\mathrm{xx}}$ about COR $\left(\mathrm{kg} \cdot \mathrm{m}^{2}\right)$ & 240950 & 0.3422 \\
$\mathrm{I}_{\mathrm{yy}}$ about COR $\left(\mathrm{kg} \cdot \mathrm{m}^{2}\right)$ & 287756 & 0.4266 \\
$\mathrm{I}_{\mathrm{zz}}$ about COR $\left(\mathrm{kg} \cdot \mathrm{m}^{2}\right)$ & 240369 & 0.3611 \\
\hline
\end{tabular}

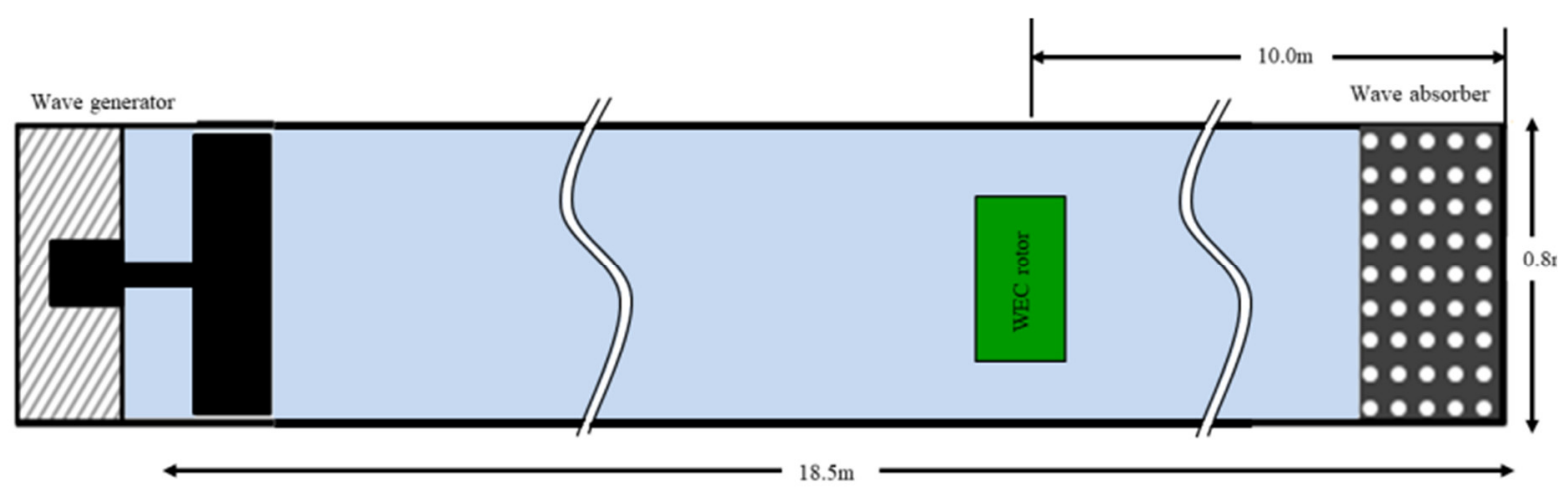

(a)

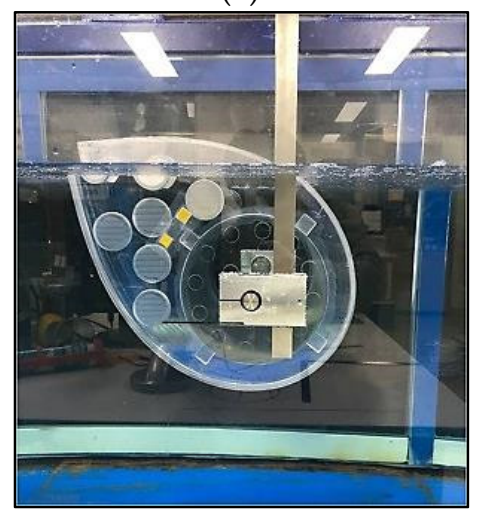

(b)

Figure 3. (a) Schematic diagram of the WEC rotor in the wave flume. (b) Experimental setup of the WEC rotor model in a flume.

\subsection{Numerical Simulations}

Similar to the experimental set up, a numerical simulation of a wave tank was performed to conduct the free decay test. The computational domain is set in the right-handed coordinate system Oxyz with the origin at the center of the WEC rotor, with $z$-axis pointing upwards and x-direction used 
for the propagation of the waves generated from the rotor (Figure 4). The dependence of the domain and mesh was carried out before arriving at the final sizes of the flow problem. The motion of the fluid flow in the computational domain is controlled by the boundary conditions (Figure 4). The initial pressure and velocity were set to the hydrostatic pressure and inlet velocity.

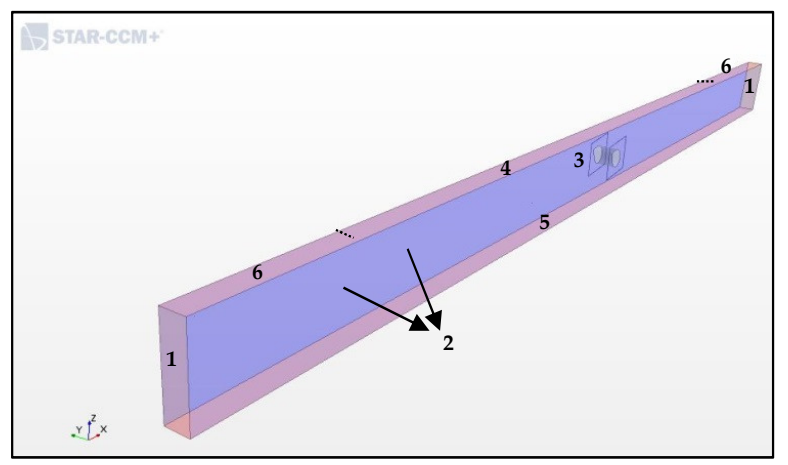

1. Outlet (Pressure outflow)

2. Symmetry walls

3. Body (Wall with no-slip condition)

4. Top (Velocity inlet with zero $\mathrm{m} / \mathrm{s}$ acts like infinite air outlet)

5. Bottom (Velocity inlet with zero $\mathrm{m} / \mathrm{s}$ )

6. Damping zone

Figure 4. Computational domain with initial boundary conditions.

The two phase free surface wave generation flow problem is not available for 2D simulations in the STAR-CCM+, in the present investigation; a quasi 2D simulation with a smaller number of elements in the y-direction was implemented. The flow problem was solved by using the continuity, momentum and turbulent k-epsilon model without modifying the default settings. Volume of fluid (VOF) was used to track the free surface in the various fluid phases and with all of the phases sharing the velocity and pressure fields. The pitch response was obtained by an overset mesh technique that is also known as overlapping meshes. Two separate regions were created, with one corresponding to the background region and another surrounding the WEC rotor (acting as an overset), and the required information is exchanged between the two regions through the overlapping cells. In the present study, linear data interpolation was used to couple the solution in the overset and background regions (see [27]). To reduce the wave reflections from both ends of the computational domain, VOF damping is enabled. The analysis was carried out with a second-order implicit unsteady time integration with five inner iterations for the convergence of the residual values. Dynamic fluid body interaction (DFBI) with fixed center of rotation about the origin in a single degree of freedom was used.

The computational domain was from $-10 \mathrm{~m}$ to $+10 \mathrm{~m}$ in the $\mathrm{x}$-direction, from $-0.1689 \mathrm{~m}$ to $+0.1689 \mathrm{~m}$ in the $\mathrm{y}$-direction and from $-0.6 \mathrm{~m}$ to $+0.6 \mathrm{~m}$ in the $\mathrm{z}$-direction. The element sizes were changed from $0.2 \mathrm{~m}$ to $0.003 \mathrm{~m}$ in the $\mathrm{x}$-direction and from $0.05 \mathrm{~m}$ and $0.2 \mathrm{~m}$ to $0.003 \mathrm{~m}$ for the $\mathrm{y}$ - and $\mathrm{z}$-directions. The viscous boundary layer $\left(\mathrm{y}^{+}\right)$around the rotor is approximately 0.9 corresponding to $0.0002 \mathrm{~m}$, whereas the recommended value should be less than 5 in order to capture the viscous effects. The domain was defined into several parts with different mesh sizes. A fine dense mesh is used where the flow variables have large gradients, i.e., at the free surface and at the rotor. The domain is discretized into small cells with a trimmed cell mesher, with the base size of $0.4 \mathrm{~m}$ in the background region and $0.003 \mathrm{~m}$ base size in the overset region defined to produce a high-quality grid consisting predominantly of hexahedral elements in the computational domain; a surface remesher was selected to generate the mesh around the rotor and the prism layer for treating the boundary layer. A prism layer with the thickness of $0.003 \mathrm{~m}$ with five as the number of the elements is selected. The total number of the cells is approximately 0.54 million in the background region and 0.83 million in the overset region. Figure 5 shows the discretized domain in and around the WEC rotor. A damping zone with the length of $4 \mathrm{~m}$ was enabled on both sides of the computational domain.

An initial displacement of $10^{\circ}$ was applied to the body. A displaced position of the WEC rotor is observed in Figure 6. Except for the fourth peak, all of the other peaks are very close to agreement with less than one percent with the experimental data as given in Table 3. The obtained pitch damping coefficient deviates by approximately four percent from the experimental result. 


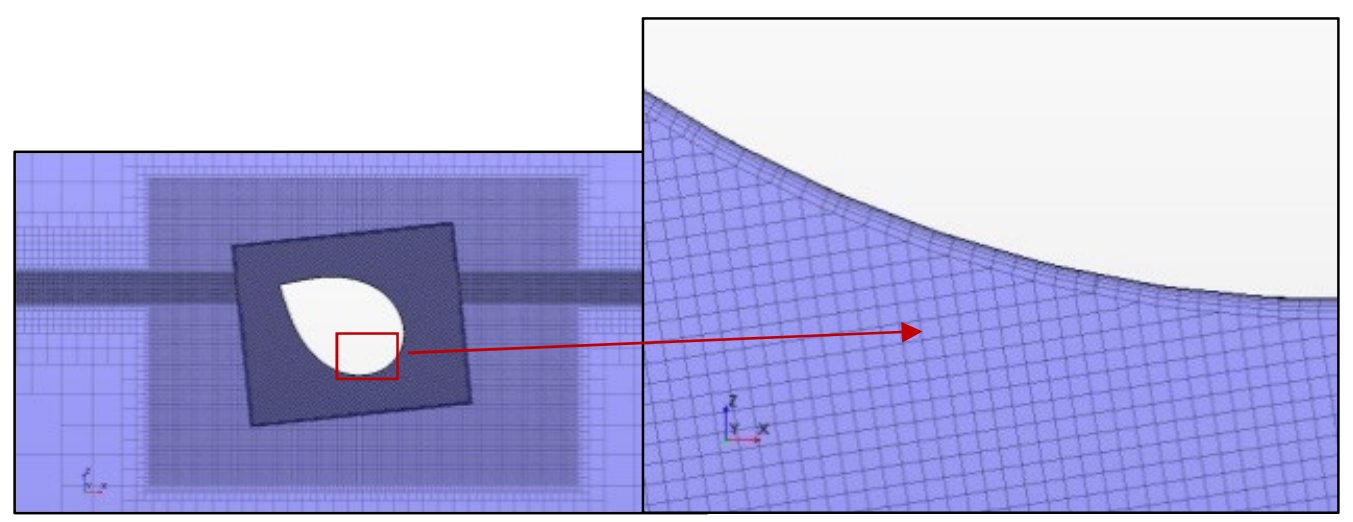

Figure 5. Initial mesh generation over the computational domain.

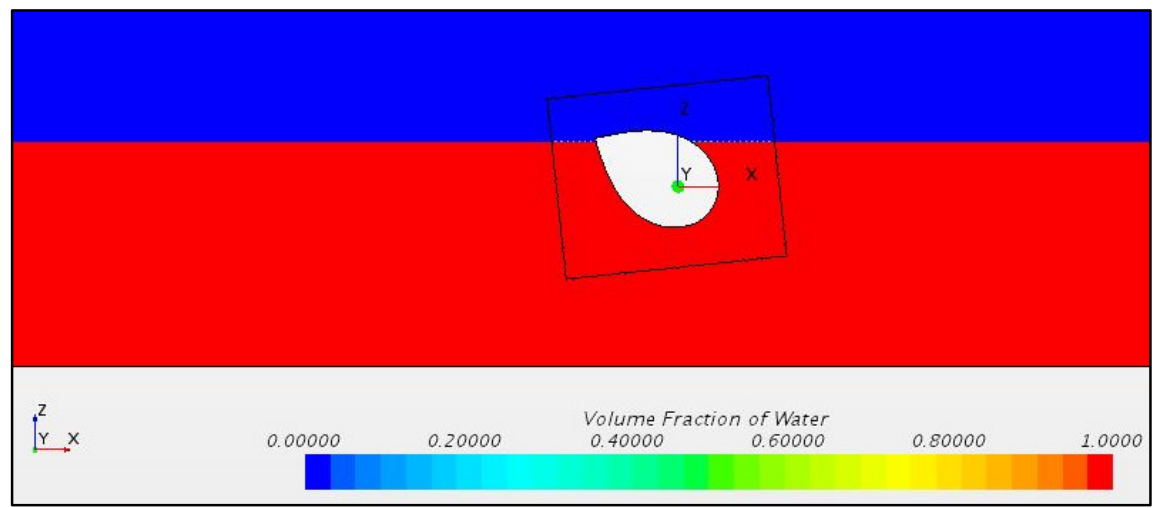

Figure 6. Initial position of the WEC rotor represented in the volume of fluid.

Table 3. Comparison of the free decay test numerical simulations and experimental data for the WEC rotor.

\begin{tabular}{cccc}
\hline Description & & Experiment & Numerical \\
\hline & $Z_{a_{i}}$ & 9.999 & 9.886 \\
Peaks (deg) & $Z_{a_{i+1}}$ & -8.596 & -8.668 \\
& $Z_{a_{i+2}}$ & 5.153 & 5.032 \\
& $Z_{a_{i+3}}$ & -5.051 & -4.522 \\
* Damping ratio (kappa) & $Z_{a_{i+4}}$ & 2.706 & 2.715 \\
(dimensionless) & & 0.0990 & 0.0955
\end{tabular}

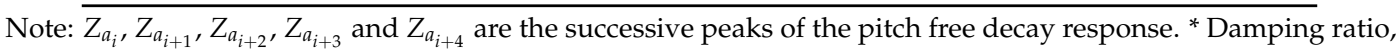
$\ln \left(\frac{Z_{a_{i}}-Z_{a_{i+1}}}{Z_{a_{i+2}}-Z_{a_{i+3}}}\right)$.

Retaining similar CFD settings that have been carried out for model study, a further analysis based on prototype WEC rotor was performed. The computational domain size with the dimensions of $400 \mathrm{~m} \times 10 \mathrm{~m} \times 24 \mathrm{~m}$ was chosen. The water surface level region around the WEC (overset region), and the overlapping regions are fairly fine with an absolute value of $2 \%$ of the base size of $5.0 \mathrm{~m}$, and refinements were made with an anisotropic trimmer to independently control the size of the cells in the $x, y$ and $z$ directions to obtained proper mesh aspect ratios in the vicinity of the mentioned regions (Figure 7). For temporal discretization, second-order unsteady implicit scheme with a time step size of $0.005 \mathrm{sec}$ for 10 inner iterations was used. VOF damping length of $100 \mathrm{~m}$ on both sides was used. 


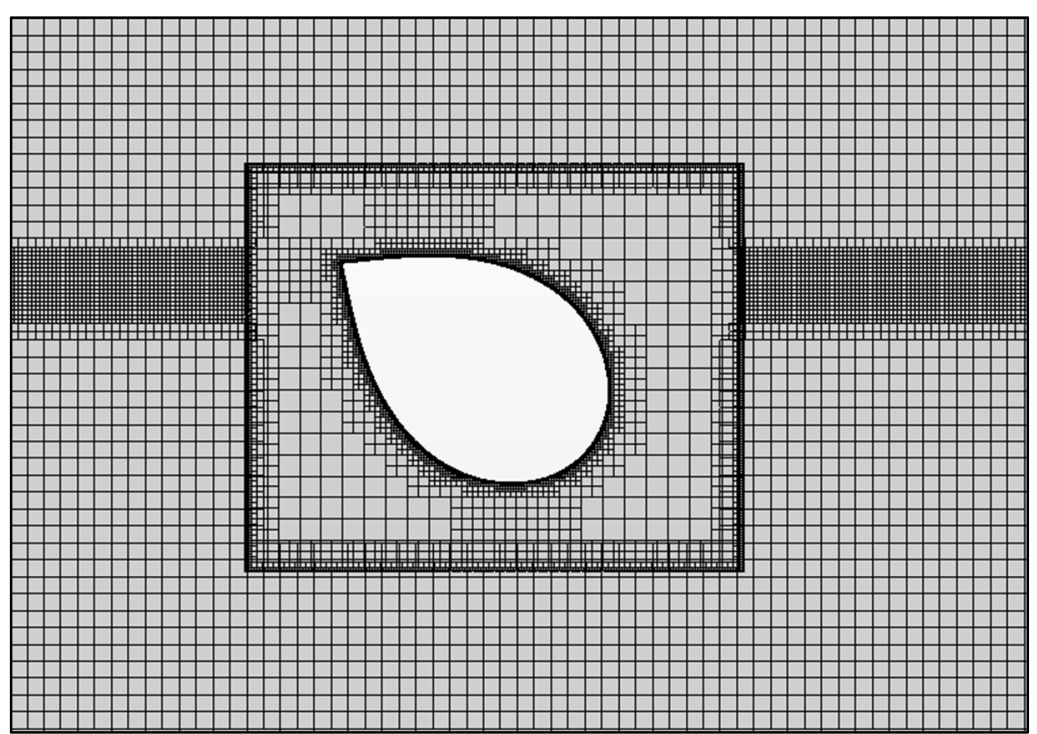

Figure 7. WEC rotor geometry with mesh in and around at the rest position.

In the energy method, the first task is to find a proper form of an approximating function that would not omit the nonlinearities of the decay time history. For this purpose, the curve fitting tool with 'Fourier' option in MATLAB [28] was used in the present study. The free decay curve was specified as the arguments to the curve fitting tool (cftool) function. The accuracy of the fitted curve can be examined based on the goodness-of-fit statistics and confidence intervals of the fitted coefficients. The goodness-of-fit helps to determine the qualification of the curve fits the input data and the confidence intervals of the coefficients describe their precision. This curve fitting provides $95 \%$ confidence bounds for the fitted coefficients. The pitch free decay response for the rotors I and II obtained using CFD are compared with the estimated free decay curve based on the energy method by the linear and linear plus quadratic moments are shown in Figure 8.

(a)
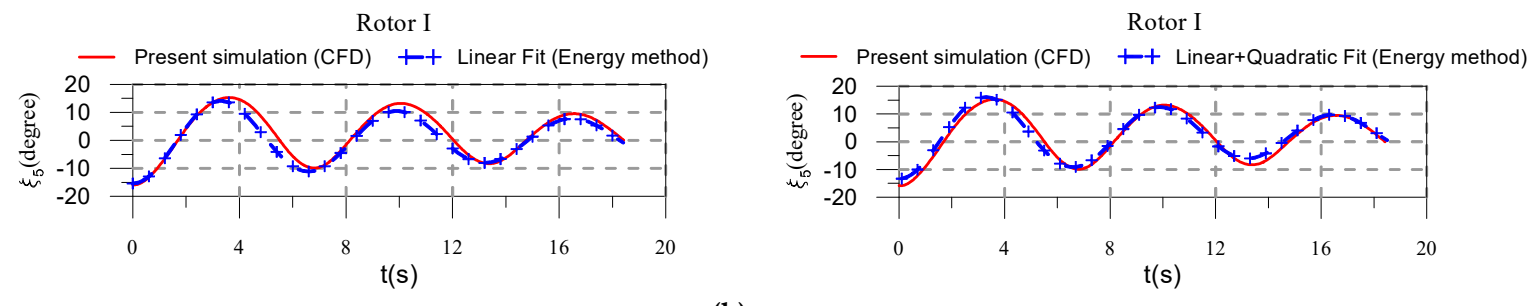

(b)
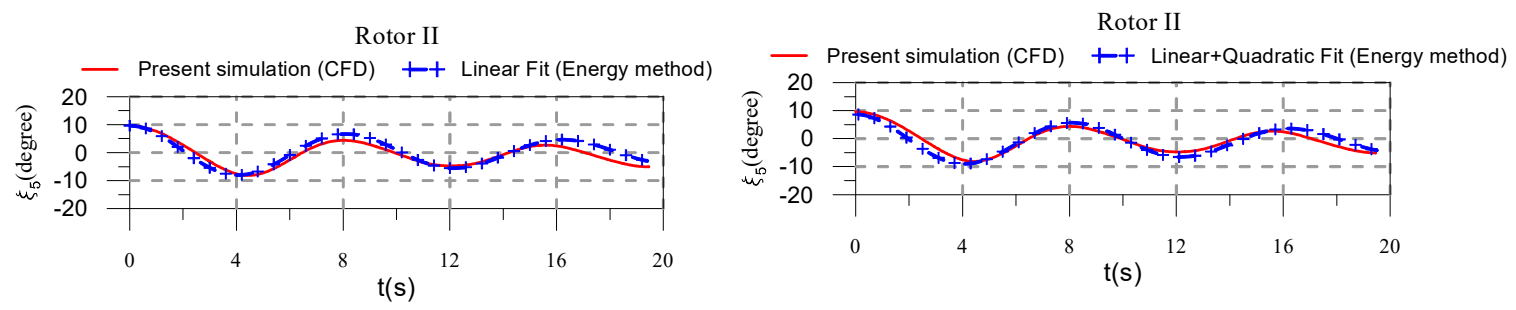

Figure 8. (a) Rotor I and (b) Rotor II; comparison of CFD and energy-based linear and linear plus quadratic free decay estimation method for different rotors.

The computed free decay data provides the total damping moment and the respective viscous damping moment was obtained by subtracting the radiation damping moment from the linear potential flow theory. Table 4 gives the estimated viscous damping coefficient values based on the linear, linear plus quadratic and linear equivalent damping moments for both of the rotors. The viscous damping 
moment coefficients obtained based on Equation (A2) (see Appendix A) contain only the leading first-order harmonic in the linear viscous damping moment (Equation (A8)) whereas the linear plus quadratic damping moment (Equation (A9)) contains the linear combination of first- and second-order harmonics. An examination of the magnitudes of these quantities (Table 4) shows that for linear damping they are almost 100 times and 25 times higher than the leading first-order harmonics for rotors I and II, respectively. The magnitude of the second-order harmonic is large for both rotors when compared to the first order harmonic. This clearly shows that the quadratic damping plays a major role in the linear plus quadratic damping. However, comparison of the linear viscous damping and linear equivalent viscous damping moments shows that the estimated viscous damping moment is of the same order of magnitude for both rotors. The predicted viscous damping values (Table 4) for the Rotor II are 5-6 times higher than those for Rotor I for linear and linear equivalent damping, respectively. The agreement between the free decay curves from CFD and the energy method appears to be good for linear damping and better for linear plus quadratic damping, as shown in Figure 8.

Table 4. Estimated pitch damping moment coefficients from the free decay tests using the energy method.

\begin{tabular}{ccccc}
\hline & & Damping Moment Coefficients & Rotor I & Rotor II \\
\hline Linear & & $B 1_{55^{\text {vis }}}(\mathrm{N} \cdot \mathrm{s} / \mathrm{m})$ & 10600 & 52210 \\
Linear plus quadratic & Linear & $B 1_{55^{\text {vis }}}(\mathrm{N} \cdot \mathrm{s} / \mathrm{m})$ & 105.1 & 2086 \\
Linear equivalent & Quadratic & $B 2_{55^{\text {vis }}}\left(\mathrm{N} \cdot \mathrm{s}^{2} / \mathrm{m}^{2}\right)$ & 1194 & 11080 \\
& & $B_{55(\mathrm{eq})}{ }^{\text {vis }}(\mathrm{N} \cdot \mathrm{s} / \mathrm{m})$ & 14100 & 87644 \\
\hline
\end{tabular}

\section{Results and Discussion}

The hydrodynamic coefficients obtained from WAMIT, viscous damping moment from the energy method and PTO damping estimated using Equation (5) were used to solve the equation of motion of the WEC rotor (Equation (3)). The pitch motion of the WEC rotors is calculated using the linear and linear plus quadratic damping moment coefficients. The predicted WEC rotor pitch motions are shown in Figure 9a,b.

For both rotors, the effect of viscous damping is significant compared to the results obtained without viscous damping. Additionally, a shift in the peak periods of the pitch response to smaller values is observed with the incorporation of linear viscous damping that is equal to approximately $1 \%$ and $2 \%$ for Rotors I and II, respectively. The effect is less than $1 \%$ for the comparison between the linear and linear plus quadratic viscous damping. The variation of non-dimensional capture width for both rotors as a function of wave frequency is shown in Figure 10. The estimated maximum capture width without viscous damping is almost close to one for Rotor II and is higher than one for the Rotor I. Significant reduction in the capture width is observed when viscous damping is included and is marginally reduced when linear plus quadratic damping is included when compared to the linear viscous damping. The corresponding optimal extracted average power estimated using Equation (4b) in regular waves with linear viscous damping is $9 \mathrm{~kW}$ and $14 \mathrm{~kW}$ for Rotors I and II, respectively, corresponding to the reduction by $6 \%$ and $15 \%$ with linear plus quadratic damping.

The pitch response in waves corresponding to the real sea environment was obtained using the JONSWAP spectrum. The spectrum includes 600 equally spaced frequencies, ranging from $0.1 \mathrm{rad} / \mathrm{s}$ to $3.0 \mathrm{rad} / \mathrm{s}$ with $\Delta \omega=0.005 \mathrm{rad} / \mathrm{s}$. The wave spectrum with peakedness factor $\gamma=2.2$, significant wave height $\left(H_{1 / 3}\right)$ of $2.0 \mathrm{~m}$ and peak period $\left(T_{p}\right)$ of $6.65 \mathrm{~s}$ was used. The square-root of the power spectral response (see Equation (6)) was obtained from the mean optimal power by the sea spectrum with a linear assumption as shown by solid dashed lines in Figure 11. 


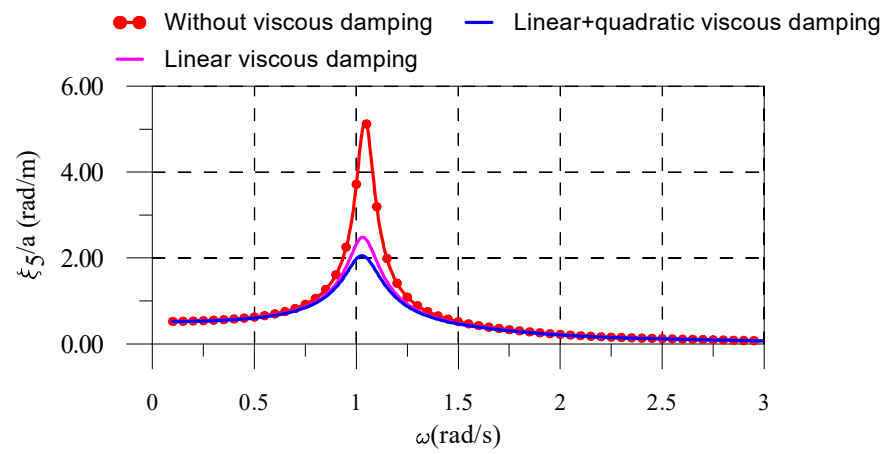

(a)

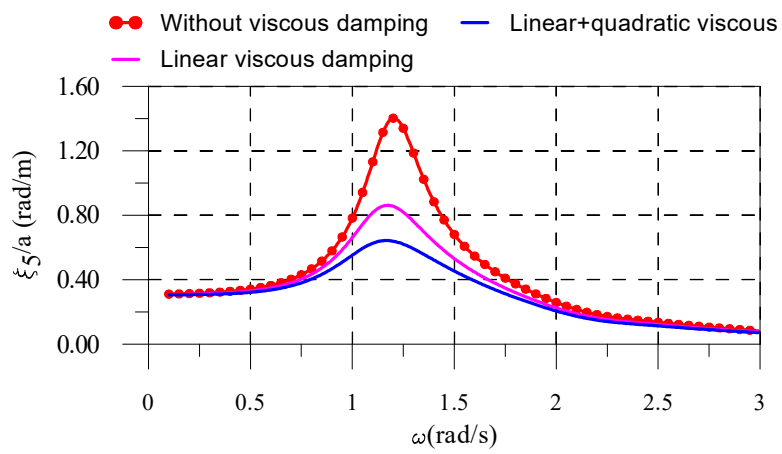

(b)

Figure 9. (a) Rotor I (b) Rotor II; variation of the pitch response of the different rotors as a function of the wave frequency.

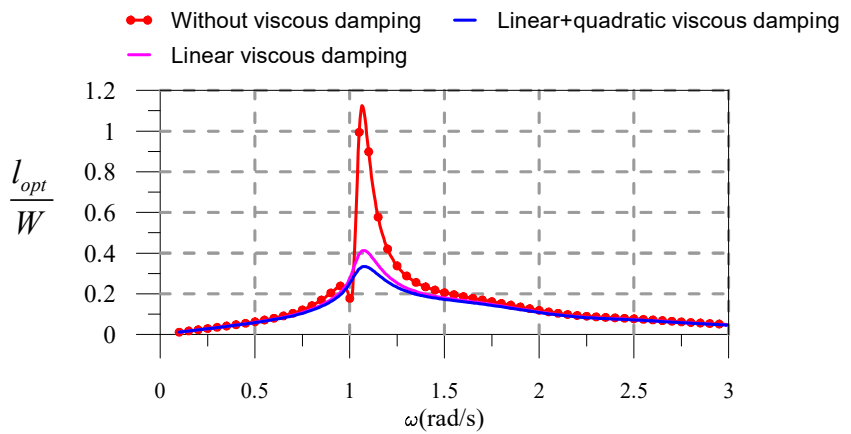

(a)

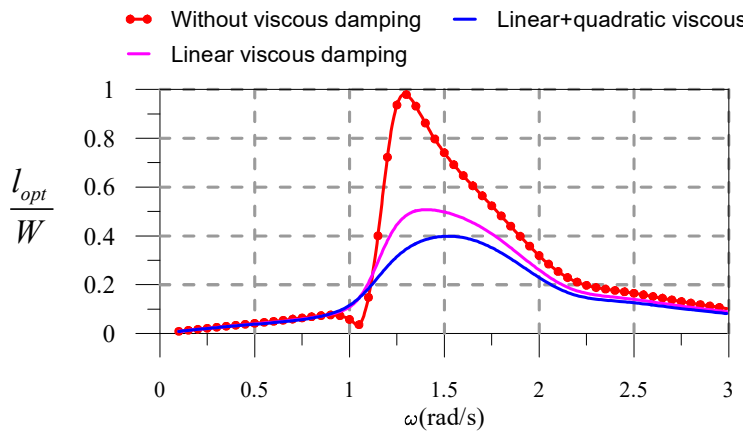

(b)

Figure 10. (a) Rotor I (b) Rotor II; Variation of non-dimensional capture width of the different rotors as a function of the wave frequency; ' $W$ ' is the width of the WEC rotor. 


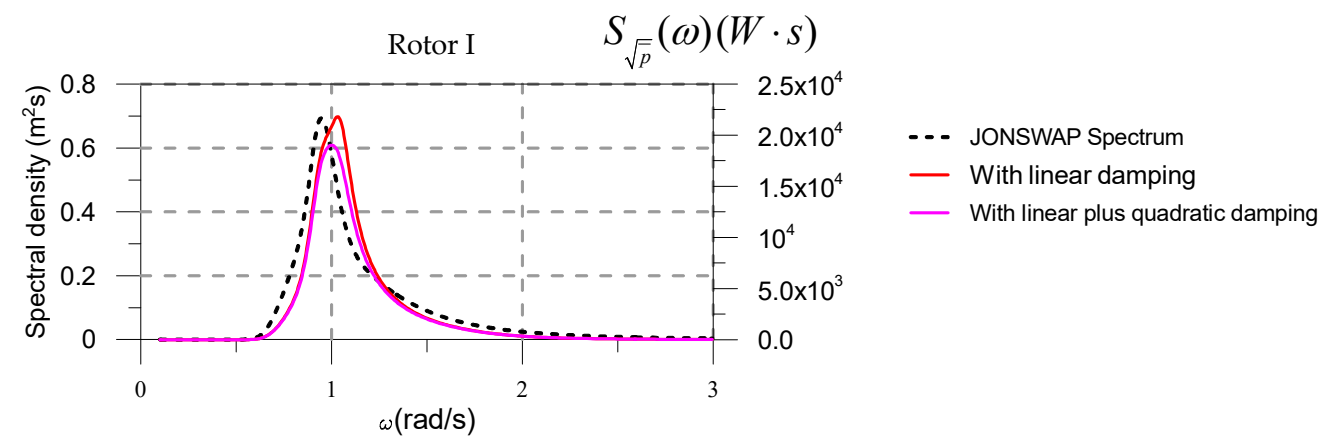

(a)

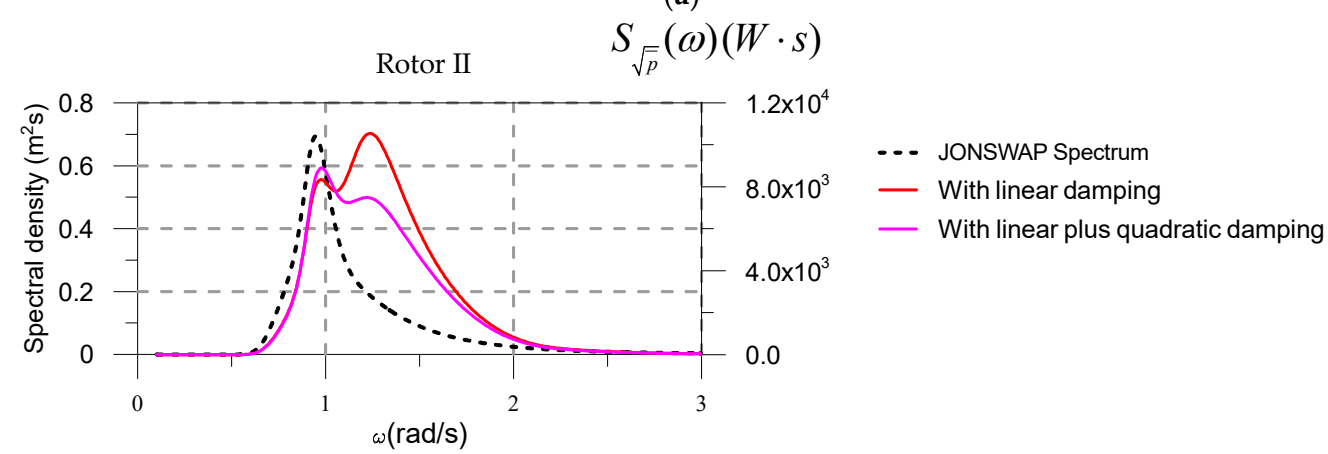

(b)

Figure 11. (a) Rotor I (b) Rotor II; Spectrum; and the square-root of power spectral response of the different rotors as a function of wave frequency.

Two prominent peaks are observed in the power spectral response (Figure 11b) for Rotor II, where one peak corresponds to the peak wave frequency of the sea state and another peak is near the natural frequency of the WEC rotor. With the variation in the peak frequencies, a reduction in the peak power is observed. The square-root of the average extracted power $\left(\bar{P}_{i r r}\right.$ by using Equation (10)) was estimated as $7.78 \mathrm{~kW}$ and $7.03 \mathrm{~kW}$ with linear and linear plus quadratic damping for Rotor I and the natural frequency is $1.03 \mathrm{rad} / \mathrm{s}$, which is closest to the wave frequency of the installation site. Similarly, for Rotor II, the square-root of the average extracted power is $7.21 \mathrm{~kW}$ and $6.11 \mathrm{~kW}$ with linear and linear plus quadratic viscous damping, respectively.

\section{Conclusions}

A frequency domain solution based on linear potential flow theory was used to obtain the hydrodynamic response of the WEC rotor and its effect due to nonlinear viscous damping is investigated. Two rotors with different depths of submergence $(0.8 \mathrm{~m}$ and $2.2 \mathrm{~m})$ and beak angle of $60^{\circ}$ are chosen for analysis. The free decay test was carried out using CFD. Linear and linear plus quadratic viscous damping moments are obtained by energy method. When linear viscous damping alone is considered, the results show good agreement with the CFD results and better agreement was obtained with linear plus quadratic viscous damping. The quadratic damping moment plays a major role, showing a large difference between the leading first order harmonic estimated from the linear and linear plus quadratic viscous damping moment; however, when the linear viscous damping and linear equivalent viscous damping moments are compared, the estimated viscous damping moment is of the same order of magnitude for both the rotors. A significant reduction in the motion amplitude is observed by adding the viscous damping. The extracted optimal average power in regular waves using linear viscous damping is $9 \mathrm{~kW}$ and $14 \mathrm{~kW}$ for Rotors I and II and it is reduced by $6 \%$ and $15 \%$ with the linear plus quadratic viscous damping, respectively. A shift in the peak periods to small values was observed with the addition of viscous damping for both rotors. The spectral analysis was carried out based on the JONSWAP spectrum and the respective square root of power spectral 
response and average extracted power were estimated. The square-root of the average extracted power for Rotor I is found be high, with the natural frequency close to the target wave frequency. The present methodology shows that better performance of WEC rotor can be obtained in regular and irregular waves only when the linear plus quadratic damping moment is considered. It would be interesting to compare the present results with the nonlinear time domain analysis where the nonlinear effects are properly taken into account; this study is now in progress.

Author Contributions: S.K.P. performed the analysis and preparation of the manuscript. Y.H.B. supervised this research and revised the manuscript. I-H.C. suggested the ideas in conceptualization of the energy method.

Funding: This work was supported by the Korea Institute of Energy Technology Evaluation and Planning (KETEP) and the Ministry of Trade, Industry \& Energy (MOTIE) of the Republic of Korea (No. 20163010071690).

Conflicts of Interest: The authors declare no conflict of interest.

\section{Appendix A. Energy Method}

The uncoupled free pitch response of the WEC rotor can be taken as:

$$
\left(J+A_{55}\right) \ddot{\xi}_{5}+B_{55} \dot{\xi}_{5}+K_{55} \tilde{\xi}_{5}=0
$$

where the damping coefficient is given by $B_{55}=B_{55}{ }^{v i s}+B_{55}{ }^{r a d}$. Higher order nonlinear damping function is given by:

$$
B_{55} \dot{\xi}_{5}=B 1_{55} \dot{\xi}_{5}+B 2_{55} \dot{\xi}_{5}\left|\dot{\xi}_{5}\right|+\ldots
$$

where the coefficients in Equation (A2) are linear, quadratic and so on. The linear and linear plus quadratic pitch motion can be expressed as:

$$
\begin{gathered}
\left(J+A_{55}\right) \ddot{\xi}_{5}+B 1_{55} \dot{\xi}_{5}+K_{55} \xi_{5}=0 \\
\left(J+A_{55}\right) \ddot{\xi}_{5}+B 1_{55} \dot{\xi}_{5}+B 2_{55} \dot{\xi}_{5}\left|\dot{\xi}_{5}\right|+K_{55} \xi_{5}=0
\end{gathered}
$$

The following steps are involved to obtain the damping from the energy method:

(1) Choosing a proper parametric form for the decay curve. Initially, various forms were tried; a 'Fourier' series up to sixth order is selected.

$$
\xi_{5}=\mathrm{a}_{\mathrm{i}-1}+\mathrm{a}_{\mathrm{i}} \cos \left(\omega_{1} \mathrm{t}_{\mathrm{i}}\right)+\mathrm{b}_{\mathrm{i}} \sin \left(\omega_{1} \mathrm{t}_{\mathrm{i}}\right) \text { where } i=1, \ldots, 6
$$

(2) The total energy of the WEC rotor can be given by Equation (A6):

$$
V(t)=\frac{1}{2}\left(J+A_{55}\right) \dot{\xi}_{5}^{2}+\int_{0}^{t} K_{55}\left(\xi_{5}\right) \dot{\xi}_{5} d t
$$

Equation (A6) contains first derivatives $\left(\dot{\xi}_{5}\right)$, these are obtained by using identified decay curve (Equation (A5)). Equation (A7) shows that the energy loss is equal to the energy dissipated in damping:

$$
V\left(t_{i+1}\right)-V\left(t_{i}\right)=-\int_{t_{i}}^{t_{i+1}} B_{55}\left(\xi_{5}\right) \xi_{5} d t
$$

where $t_{i}$ and $t_{i+1}$ are the starting and end of the pitch decay curve. The damping moment is defined in a form of linear and linear plus quadratic terms as:

$$
B_{55}\left(\dot{\xi}_{5}\right)=B 1_{55} \dot{\xi}_{5}
$$




$$
B_{55}\left(\dot{\xi}_{5}\right)=B 1_{55} \dot{\xi}_{5}+B 2_{55} \dot{\xi}_{5}\left|\dot{\xi}_{5}\right|
$$

(3) The method of least-squares is used to obtain the damping moment coefficients from Equations $\mathrm{A}(10)$ and $\mathrm{A}(11)$ :

$$
\begin{gathered}
V\left(t_{i+1}\right)-V\left(t_{i}\right)=-\int_{t_{i}}^{t_{i+1}} B 1_{55} \xi_{5}^{2} d t \\
V\left(t_{i+1}\right)-V\left(t_{i}\right)=-\int_{t_{i}}^{t_{i+1}}\left(B 1_{55} \xi_{5}^{2}+B 2_{55} \xi_{5}^{2}\left|\xi_{5}\right|\right) d t
\end{gathered}
$$

(4) Finally, along with the damping moment coefficients, the equation of motion (Equation (A1)) is solved by using the Runge-Kutta fourth order method.

\section{References}

1. Bhattacharyya, R.; McCormick, M.E. Wave Energy Conversion; Elsevier: Oxford, UK, 2003.

2. Cruz, J. Ocean Wave Energy: Current Status and Future Perspectives; Springer Science and Business Media: Berlin, Germany, 2007.

3. Pecher, A.; Kofoed, J.P. Handbook of Ocean Wave Energy; Springer International Publishing: Cham, Switezerland, 2017.

4. Salter, S.H. Wave power. Nature 1974, 249, 720-724. [CrossRef]

5. Mei, C.C. Power extraction from water waves. J. Ship Res. 1976, 20, 63-66.

6. Pecher, A.; Kofoed, J.P.; Larsen, T. The extensive R\&D behind the Weptos WEC. Renew. Energ. Offshore 2015, $1,351$.

7. Salter, S.; Jeffrey, D.C.; Taylor, J.R.M. First Year Interim Report on Edinburgh Wave Power Project: Study of Mechanisms for Extracting Power from Sea Waves; University of Edinburgh: Edinburgh, UK, 1975.

8. Jeffrey, D.C.; Richmond, D.J.E.; Salter, S. Second Year Interim Report on Edinburgh Wave Power Project: Study of Mechanisms for Extracting Power from Sea Waves; University of Edinburgh: Edingburg, UK, 1976.

9. Pecher, A.; Kofoed, J.P.; Larsen, T.; Marchalot, T. Experimental study of the Weptos wave energy converter. In Proceedings of the ASME 31st International Conference on Ocean, Offshore and Arctic Engineering, Rio de Janeiro, Brazil, 1-6 July 2012; pp. 525-534.

10. Chakrabarti, S.K. Handbook of Offshore Engineering; Elsevier: Amsterdam, The Netherlands, 2005; p. 1.

11. Wu, J.; Yao, Y.; Li, W.; Zhou, L.; Göteman, M. Optimizing the performance of solo duck wave energy converter in tide. Energies 2017, 10, 289. [CrossRef]

12. Poguluri, S.K.; Bae, Y.H. A study on performance assessment of WEC rotor in the Jeju western waters. Ocean Syst. Eng. 2018, 8, 361-380.

13. Dalzell, J.F. A Note on the Form of Ship Roll Damping; United States Defense Technical Information Center: Fort Belvir, VA, USA, 1978; p. 22.

14. Roberts, J.B.; Spanos, P.D. Random Vibration and Statistical Linearization; Courier Corporation: Chelmsford, MA, USA, 2003.

15. Mathisen, J.B.; Price, W.G. Estimation of ship roll damping coefficients. R. Inst. Nav. Archit. Trans. 1985, 127, 13.

16. Poguluri, S.K.; Bae, Y.H.; Cho, I.H. Effect of viscous damping on the performance of a pitch-type WEC rotor. In Proceedings of the 28th International Ocean and Polar Engineering Conference, Sapporo, Japan, 10-15 June 2018.

17. Bass, D.W.; Haddara, M.R. Nonlinear models of ship roll damping. Int. Shipbuild. Prog. 1988, 35, 5-24.

18. Roberts, J.B. Estimation of nonlinear ship roll damping coefficients from free decay data. J. Ship Res. 1985, 29, $411-427$.

19. Zhang, J. Design and Hydrodynamic Performance of Trimaran Displacement Ships. Ph.D. Thesis, University of London, London, UK, 1997.

20. Sun, L.P.; Sun, W.B. Parameter identification of the non-linear rolling damping based on PLS regression technique. Adv. Mater. Res. 2013, 779, 675-679. [CrossRef] 
21. Uzunoglu, E.; Soares, C.G. Automated processing of free roll decay experimental data. Ocean Eng. 2015, 102, 17-26. [CrossRef]

22. Lloyd, A.R.J.M. Seakeeping: Ship Behaviour in Rough Weather; Ellis Horwood: Hemel Hempstead, UK, 1989.

23. Falzarano, J.; Somayajula, A.; Seah, R. An overview of the prediction methods for roll damping of ships. Ocean Syst. Eng. 2015, 5, 55-76. [CrossRef]

24. Falnes, J. Ocean Waves and Oscillating Systems: Linear Interactions including Wave-Energy Extraction; Cambridge University Press: Cambridge, UK, 2002.

25. Mei, C.C.; Stiassnie, M.; Yue, D.K.P. Theory and Applications of Ocean Surface Waves: Nonlinear Aspects; World Scientific: McGraw Hil, Singapore, 2005.

26. Ghadimi, P.; Bandari, H.P.; Rostami, A.B. Determination of the heave and pitch motions of a floating cylinder by analytical solution of its diffraction problem and examination of the effects of geometric parameters on its dynamics in regular waves. Int. J. Appl. Math. Res. 2012, 1, 611-633. [CrossRef]

27. Hadzic, H. Development and Application of Finite Volume Method for the Computation of Flows Around Moving Bodies on Unstructured, Overlapping Grids. Ph.D. Thesis, Technische Universität Hamburg, Hamburg, Germany, 2006.

28. MatLab. The Language of Technical Computing. The MathWorks, Inc. Available online: http://www.mathworks. com (accessed on 11 February 2019).

(C) 2019 by the authors. Licensee MDPI, Basel, Switzerland. This article is an open access article distributed under the terms and conditions of the Creative Commons Attribution (CC BY) license (http://creativecommons.org/licenses/by/4.0/). 